\title{
A Laminar Vortex Interacting with a Premixed Flame: Measured Formation of Pockets of Reactants
}

\author{
WILLIAM L. ROBERTS and JAMES F. DRISCOLL \\ Department of Aerospace Engineering, University of Michigan, Ann Arbor, MI 48109
}

\begin{abstract}
A single toroidal, laminar vortex in created and a laminar premixed flame is propagated over the vortex in order to study the fundamental interaction process and to compare results with the numerical solutions of the Navier-Stokes equations obtained by Poinsot, Veynante, and Candel. Results quantify the degree of flame rollup, the flame perimeter increase due to wrinkling, the minimum vortex size that wrinkles the flame, and the boundary of the pocket formation (corrugated flame) regime. The measured trends indicate that viscous attenuation of the vortex by the hot, viscous products is important. The importance of flame stretch is verified; as Lewis number is varied it is found that in order to form pockets, diffusionally stable flames require a vortex strength that is three times larger than that required by the diffusionally unstable flames.
\end{abstract}

\section{INTRODUCTION}

The present study is motivated by the following research questions. First, the degree of strain, curvature, and quenching exerted on a flame by each vortex in a turbulent flowfield is not known. There is abundant information about a steady, planar laminar flame in a strain field near a stagnation point [1]. However, the planar stagnation point flame properties may not adequately simulate local conditions near strained turbulent flames because turbulent vortices introduce the additional effects of flame curvature and a timevarying strain field [2] as the vortex passes over the flame. The present study of a single vortex passing through a flame can help to relate the theory for a planar stagnation point flame to local conditions in a turbulent flame.

The second research question relates to the formation of pockets, or islands, of unburned reactants. The vortex strength required to form pockets and therefore cause the flame to enter the "corrugated flame" regime is not known. These pockets of reactants may not burn completely, which can cause excessive levels of carbon monoxide or unburned hydrocarbons under conditions of relatively low Damkohler number, i.e., high turbulence level or excessive exhaust gas recirculation. The present results quantify the boundaries of the corrugated regime for a single vortex and allow for the assessment of two recent numerical simulation methods that predict the formation of pockets. Poinsot et al. [3] and Rutland et al. [4] solve the Navier Stokes equations numerically for a laminar flame-vortex interaction. Kwon et al. [5] and Wu et al. [6] use a flame interface simulation and also predict the formation of pockets. Other simulations of the flame-vortex interaction have been reported by Chate and Cant [7], Ashurst and McMurtry [8], and Peters and Williams [9].

The third research question concerns the minimum diameter of vortices that can wrinkle a premixed flame. Peters [10] describes a minimum vortex diameter, which he denotes the Gibson scale. Poinsot et al. [3] predict that a different vortex scale is the smallest that wrinkles the flame. The results of the present measurements, described below, indicate that the physical trends predicted by Poinsot et al. are correct. That is, the minimum scale of wrinkling and the conditions at which pockets first form are strongly influenced by gas viscosity and flame curvature.

Consider a typical flame-vortex interaction illustrated in Fig. 1. Shown is a planar view of a toroidal vorticity layer. The rotational velocity of the vortex has two effects. First, there is a hydrodynamic effect caused by the vortex velocity field that wrinkles the flame and can cause pockets even with no stretch or viscosity effects. The vortex has a component of velocity on the centerline that is towards the flame, which retards flame motion on the centerline; the vortex velocity far from the centerline has a component in the direc- 


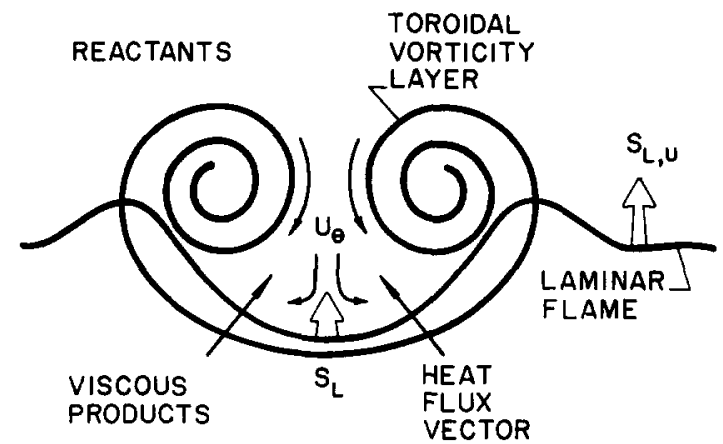

Fig. 1. Schematic of the laminar flame propagating upward over the toroidal vortex. On centerline, the flame experiences positive strain (similar to a stagnation point flow) which decreases $S_{L}$, and experiences negative curvature (similar to an inverted Bunsen burner) which increases $S_{L}$ for Le $>1$.

tion of flame propagation, causing the flame to wrinkle (Fig. 1). As this wrinkle passes over the rear portion of the vortex, the rotational velocity pulls the flame towards the centerline which tends to create a pocket of unburned reactants if $U_{\theta} / S_{L}$ is sufficiently large. Secondly, the rotational velocity exerts stretch on the flame that can augment this wrinkling/pocket formation process. Flame stretch is due to the imposed strain field and to flame curvature. The stagnation point strain field near the centerline induces positive stretch and can reduce the local burning velocity for Lewis numbers exceeding unity. The curvature of the flame at the centerline induces negative stretch, which can affect the burning velocity in a manner opposite to the strain field. The flame curvature is similar to that of an inverted Bunsen burner, which is well documented [1]. Some vortex conditions for which strain dominates over curvature (and vice versa) were predicted by the numerical solutions of Poinsot et al. [3].

If one considers a time-varying property of the flame surface, such as the flame surface area $A$, we expect that the primary nondimensional parameters of importance are

$$
\begin{aligned}
\frac{A-A_{0}}{A_{0}}= & \operatorname{fen}\left(\frac{t}{d_{0} / S_{L}}, \frac{U_{\theta}}{S_{L}}, \frac{U_{z}}{S_{L}}, \frac{d_{0}}{\delta_{F}},\right. \\
& \left.\operatorname{Re}, \mathrm{Da}, \mathrm{Le}, \mathrm{Ri}, \frac{T_{P}}{T_{R}}\right),
\end{aligned}
$$

where $A_{0}$ is the initial flame surface area, $d_{0}$ is defined below to be proportional to the core-tocore distance of the vortex shown in Fig. 1, and
$S_{L}$ is the laminar burning velocity of the undisturbed reactants. $U_{\theta}$ is the maximum rotational velocity within the vortex and $U_{z}$ is the translational velocity of the vortex core with respect to the unburned gas ahead of the flame. Therefore the relative velocity between the vortex core and the undisturbed flame is $U_{z}+S_{L}$. The undisturbed flame thickness is $\delta_{F}$ and is defined as $\left(T_{\max }-T_{\min }\right) /(d T / d x)_{\max }$. Dixon-Lewis [11] has shown that $\delta_{F}$ is approximately $3.7 \alpha / S_{L}$, where $\alpha$ is the thermal diffusivity of the reactants; therefore this relation is used in the present work and was used by Poinsot et al. [3].

The effects of viscosity are introduced by the Reynolds number $U_{\theta} d_{0} / \nu_{R}$ and the temperature ratio $T_{P} / T_{R}$, which determines the viscosity of the products. The viscosity of the products is especially important in attenuating the vortex rotational velocity as the vortex passes through the flame. Flame stretch is quantified by $d_{0} / \delta_{F}$, which characterizes the flame curvature, and by the Damkohler number $\mathrm{Da}$, which equals $\left(d_{0} / \delta_{F}\right) /\left(U_{\theta} / S_{L}\right)$ and characterizes the strain field. It is noted that $\mathrm{Da}$ is not an independent parameter but is the ratio of two previously defined parameters. The Lewis number Le quantifies thermodiffusive and stretch effects; it equals the thermal diffusivity of the reactant mixture, normalized by the mass diffusivity of the deficient reactant into nitrogen. Buoyancy forces are characterized by the Richardson number $\mathrm{Ri}$, which equals $\left.\left(\left(\rho_{R}+\rho_{P}\right) / 2\right) U_{\theta}^{2}\right) /\left(\left(\rho_{R}-\rho_{P}\right) g d_{0}\right) ; g$ is the acceleration of gravity. Heat release is quantified by the ratio of the adiabatic flame temperature $T_{p}$ to the reactant temperature $T_{R}$. In the present experiments, four of the above nine parameters were varied independently; these parameters are $t /\left(d_{0} / S_{L}\right), U_{\theta} / S_{L}, d_{0} / \delta_{F}$, and Le. Values of $\mathrm{Re}, \mathrm{Da}$, and $\mathrm{Ri}$ did not vary in an independent fashion, but varied due to variations in $U_{\theta}$ and $d_{0}$.

\section{APPARATUS}

The experiment consists of a rectangular flame tube, $61 \mathrm{~cm}$ high and $11.4 \mathrm{~cm}$ on a side (Fig. 2). The front is optical quality glass, and each side has a slit covered with glass for visualization experiments utilizing a laser sheet. The bottom of the flame tube contains a spark source, a fan for mixing the fuel-air charge, and a fuel delivery 


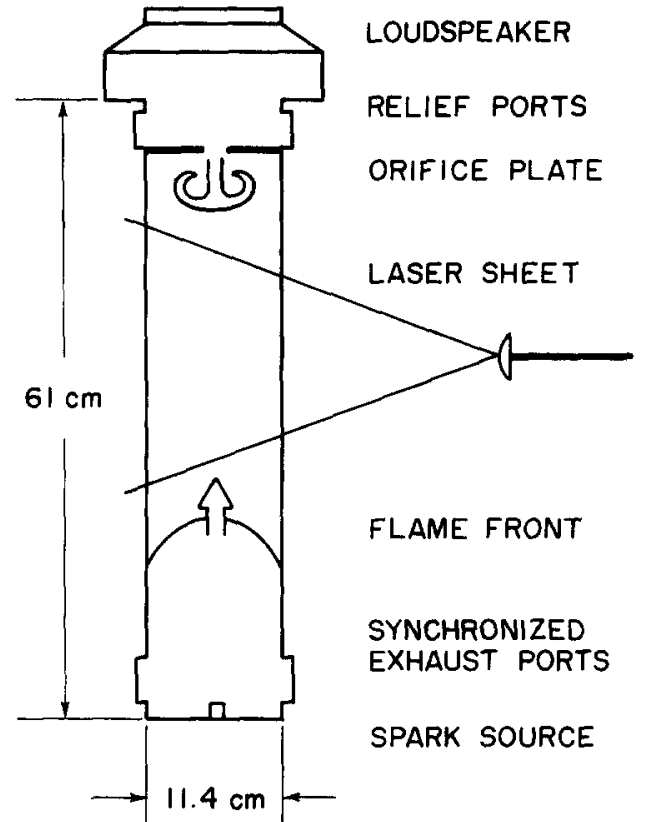

Fig. 2. Apparatus used to interact the laminar flame and laminar vortex.

tube. The top is sealed by either a $20.3-\mathrm{cm}$-diameter or $10.2-\mathrm{cm}$-diameter loudspeaker. Located $55.9 \mathrm{~cm}$ from the bottom of the flame tube is an interchangeable sharp-edged orifice plate that separates the upper chamber in Fig. 2 from the lower chamber. The orifice diameter is designated $d_{0}$ and varies from 0.64 to $5.1 \mathrm{~cm}$.

The desired amount of fuel is injected into the lower chamber by decreasing the pressure in a fuel reservoir, having known volume, by a predetermined amount. Fuel is injected into the upper chamber by the same procedure, ensuring that the mixture ratios of both chambers are equal. The fuel and air are mixed with the fan and the resulting turbulence dissipates after an appropriate settling time. Experiments were conducted using either methane or propane at an equivalence ratio $\phi$ of 0.6 . The mixture is ignited using a standard automotive sparkplug. The hot products are exhausted during the interaction through solenoid operated ports $(2.5 \times 7.6 \mathrm{~cm})$ near the bottom of the flame tube and pressure relief valves in the upper chamber.

The undisturbed laminar burning velocity of methane at $\phi$ of 0.6 was determined to be $8 \mathrm{~cm} / \mathrm{s}$ using two methods. Yamaoka and Tsuji [12], Yu et al. [13], and Lewis and von Elbe [14] report this value of $S_{L}$; in addition, $S_{L}$ was measured by subtracting the gas velocity ahead of the flame $(34 \mathrm{~cm} / \mathrm{s})$, as measured using laser velocimetry, from the flame speed in laboratory coordinates $(42 \mathrm{~cm} / \mathrm{s})$, determined from VCR images. Very smooth, nonwrinkled laminar flames were achieved, providing that values of $\phi$ were less than 0.7 for methane and for propane fuels. For values of $\phi$ greater than 0.7 excessive wrinkling was caused by hydrodynamic instabilities enhanced by the pressure buildup behind the flame.

To generate a vortex ring, a voltage ramp function is applied across the coil of the loudspeaker, causing the speaker to deflect and push fluid through the orifice [15-18]. The translational velocity of the vortex core and the maximum rotational velocity were measured using laser velocimetry and were determined to be approximately equal. Both velocities were a function of the voltage applied to the speaker, the diameter of the orifice, and the pulse duration of the voltage ramp function as described in the Appendix. Rotational velocities between 2.5 and $300 \mathrm{~cm} / \mathrm{s}$ were achieved.

Mie Scattering Tomography was used to visualize the flame-vortex interaction. Micron-sized oil drops from a TSI 9301 atomizer were added to the reactants and disappeared at the flame front. An argon ion laser and a cylindrical lens were used to form a laser sheet, which was viewed using a LoCam II 16-mm camera operated at 300 frames/s with a $10-\mathrm{nm}$ interference filter to remove flame radiation.

\section{RESULTS - VISUALIZATION OF FLAME-VORTEX INTERACTIONS}

The interaction between the laminar premixed flame and the laminar vortex ring can be characterized using four distinct classifications-the no-effect regime, the wrinkled flame regime, the pocket formation regime, and the local quenching regime. The first three regimes were studied herein and are defined as follows. With no vortex present, the flame has a curvature that is convex towards the reactants due to wall boundary layers, as shown in Fig. 2. It was decided to denote the onset of wrinkling as the condition when the flame curvature changes sign, i.e., becomes concave toward the reactants on the centerline, which results in a $5 \%$ change of the flame perimeter. 


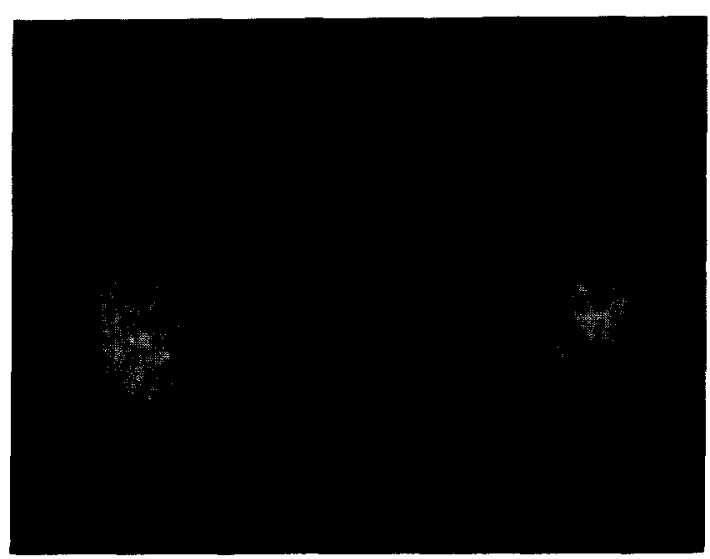

Fig. 3. A typical undisturbed vortex ring photographed using a laser light sheet and submicron oil drops. $d_{0}=5.08 \mathrm{~cm}$, $U_{\theta}=50 \mathrm{~cm} / \mathrm{s}$.

The pocket formation regime occurs if an independent pocket of reactants survives the flame passage for more than $1 / 60$ of a second. The fourth regime in which local flame quenching occurs has been studied by applying planar $\mathrm{OH}$ imaging to the present experiment and the results are reported elsewhere by Roberts et al. [19].

Figure 3 shows a typical vortex ring cross-section visualized by the laser light sheet. Oil drops were added only to the upper chamber for this image. These images were used to determine that the vortex core-to-core distance is $1.2 d_{0}$ at this downstream location, where $d_{0}$ is the orifice diameter, which is consistent with previous vortex studies [17]. Figure 4 shows a very weak vortex $\left(U_{\theta} / S_{L}=0.5\right)$ interacting with the flame front. Reactants are on top of the interface and products are on the bottom; products appear black due to the lack of oil drops which burn up in the flame front. The cores of the vortex pair are black because oil drops were not added to the upper chamber, so as to visualize the vortex core location. As can be seen, the vortex reduces the curvature, but does not wrinkle the flame front. The flame forces the vortex cores to move apart in Fig. 4, which is similar to motions that occur when a vortex pair approaches a solid boundary, which can be explained by the concept of image vortices. The flame appears to act like a solid boundary at the beginning of the interaction because the high viscosity of the products tends to damp out induced velocities in the products. The parabolic shape of the flame surface also may help to separate the vortex cores.

Figure 5 shows the interaction between the flame and a stronger vortex having $U_{\theta} / S_{L}=1.5$. This interaction results in significant wrinkling of the flame area, but no pocket is formed. In Fig. 6 the vortex strength has bee increased to $U_{\theta} / S_{L}=$ 2.75. The vortex already is strong enough to separate itself from the flame front and exist as a pocket of reactants. This pocket completely disappears $131 \mathrm{~ms}$ after any effect on the flame by the vortex is first detected. The oil droplet visualization technique cannot identify whether the border between reactants and products is a reaction zone or is locally quenched. Note that there is very little "vortex wake" in Fig. 6.

In Fig. 7 the vortex strength has been increased to $U_{\theta} / S_{L}=8.8$. The rotational velocity first pulls

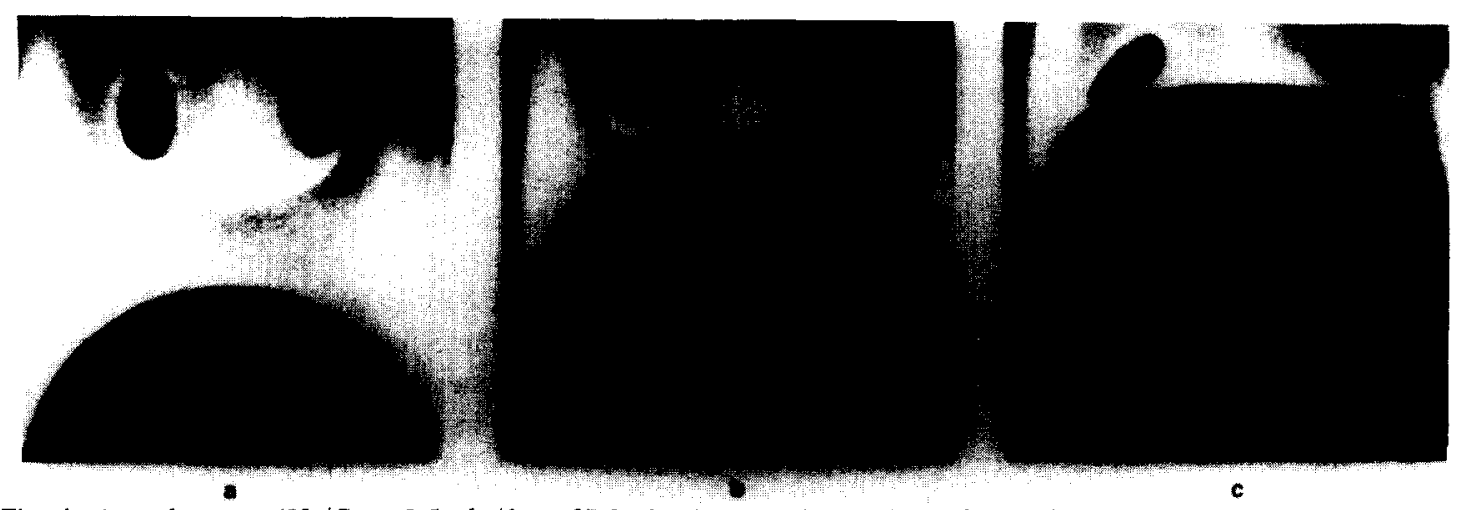

Fig. 4. A weak vortex $\left(U_{\theta} / S_{L}=0.5, d_{0} / \delta_{F}=37.2\right)$ that has no effect on flame front. The distance between vortex cores increases, similar to vortices interacting with a solid surface. Methane, $\phi=0.6, S_{L}=8 \mathrm{~cm} / \mathrm{s}, \delta_{F}=0.10 \mathrm{~cm}$. 


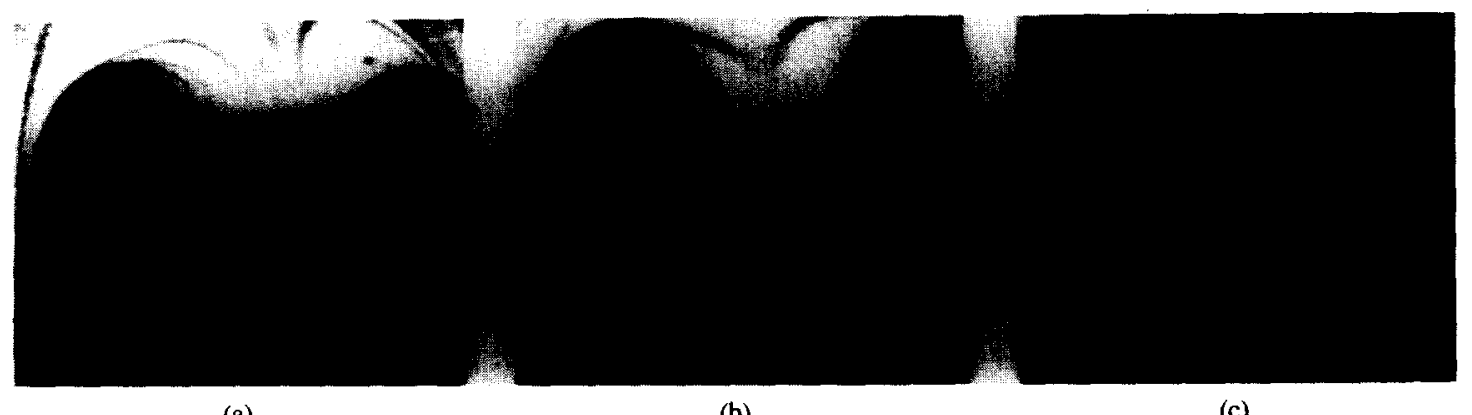

(a)

(b)

(c)

Fig. 5. Flame surface visualized in the wrinkled flame regime $\left(U_{\theta} / S_{L}=1.5, d_{0} / \delta_{F}=37.2\right.$, no pocket of reactant formed). Methane, $\phi=0.6$. Time from beginning of interaction is (a) $50 \mathrm{~ms}$, (b) $88 \mathrm{~ms}$, and (c) $96 \mathrm{~ms}$.

the flame in the vortex wake toward the centerline, which creates a narrow necking of the reactants in the wake region. The flame then enters the rear of the vortex and begins to roll up. The wake is longer than in Fig. 6 due to the increased translational velocity (which is proportional to rotational velocity). The flame rolls up and travels almost 270 degrees around the vortex. A jet of hot gas products is observed to issue upward along the centerline in Figs. $7 \mathrm{~g}-7 \mathrm{i}$ due to gas

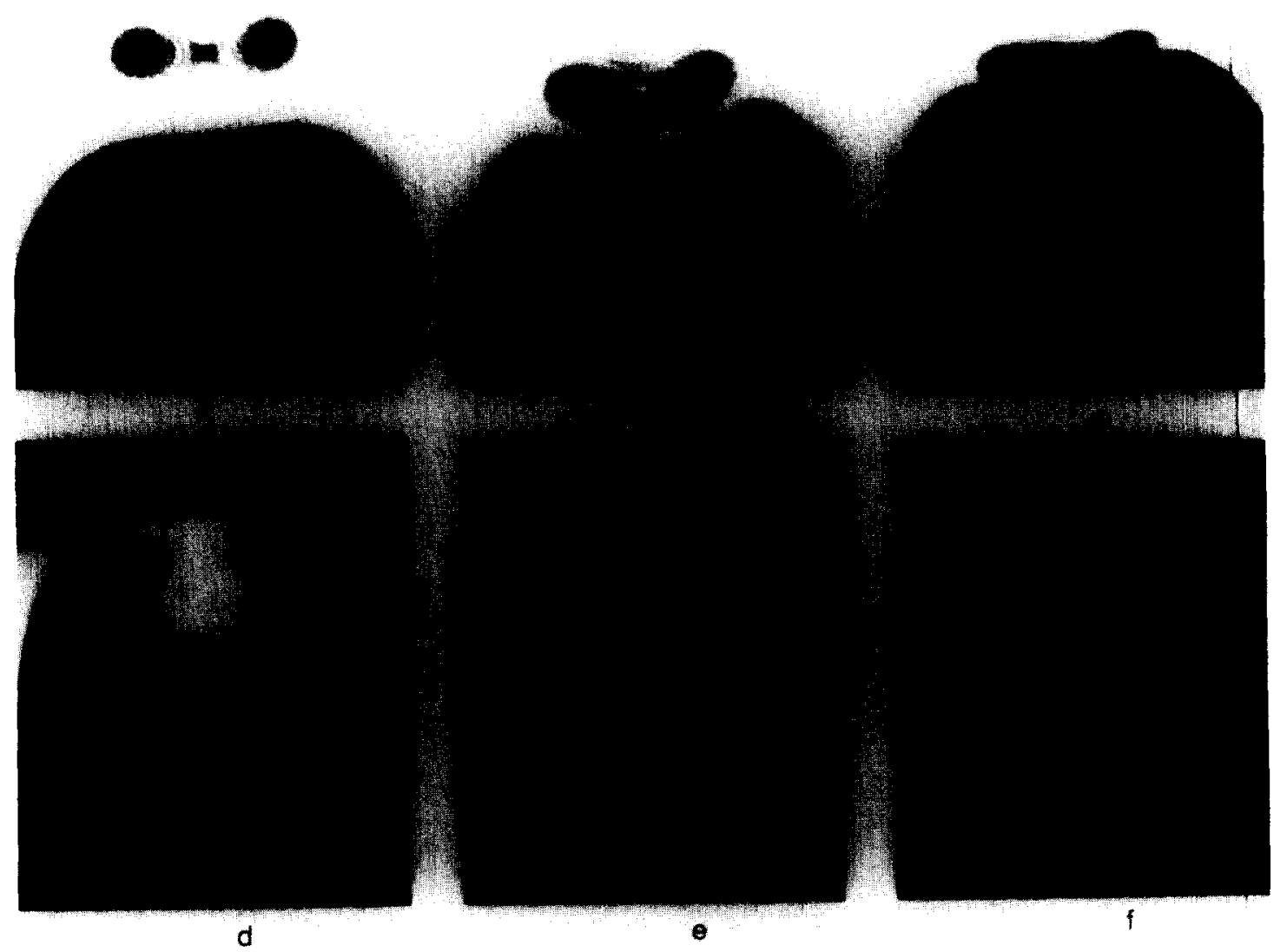

Fig. 6. Formation of pocket of reactant with no flame rollup. $U_{\theta} / S_{L}=2.75, d_{0} / \delta_{F}=37.2$, methane, $\phi=0.6$. Time: (a) 31 $\mathrm{ms}$, (b) $63 \mathrm{~ms}$, (c) $77 \mathrm{~ms}$, (d) $100 \mathrm{~ms}$, (e) $106 \mathrm{~ms}$, and (f) $111 \mathrm{~ms}$. 


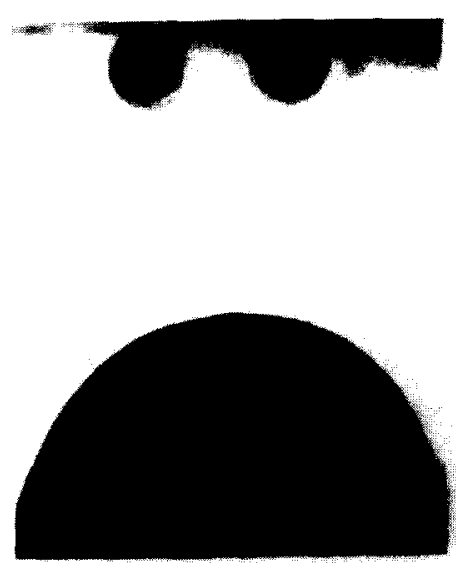

a

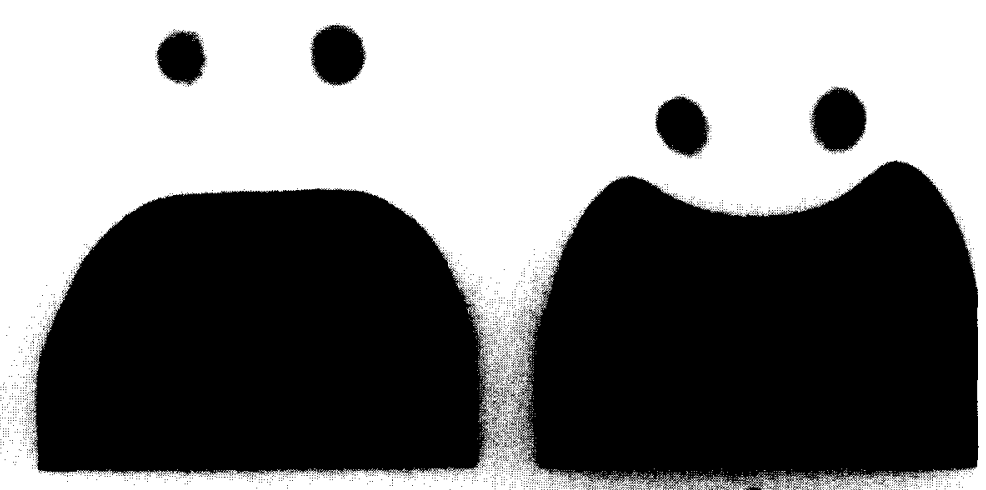

b
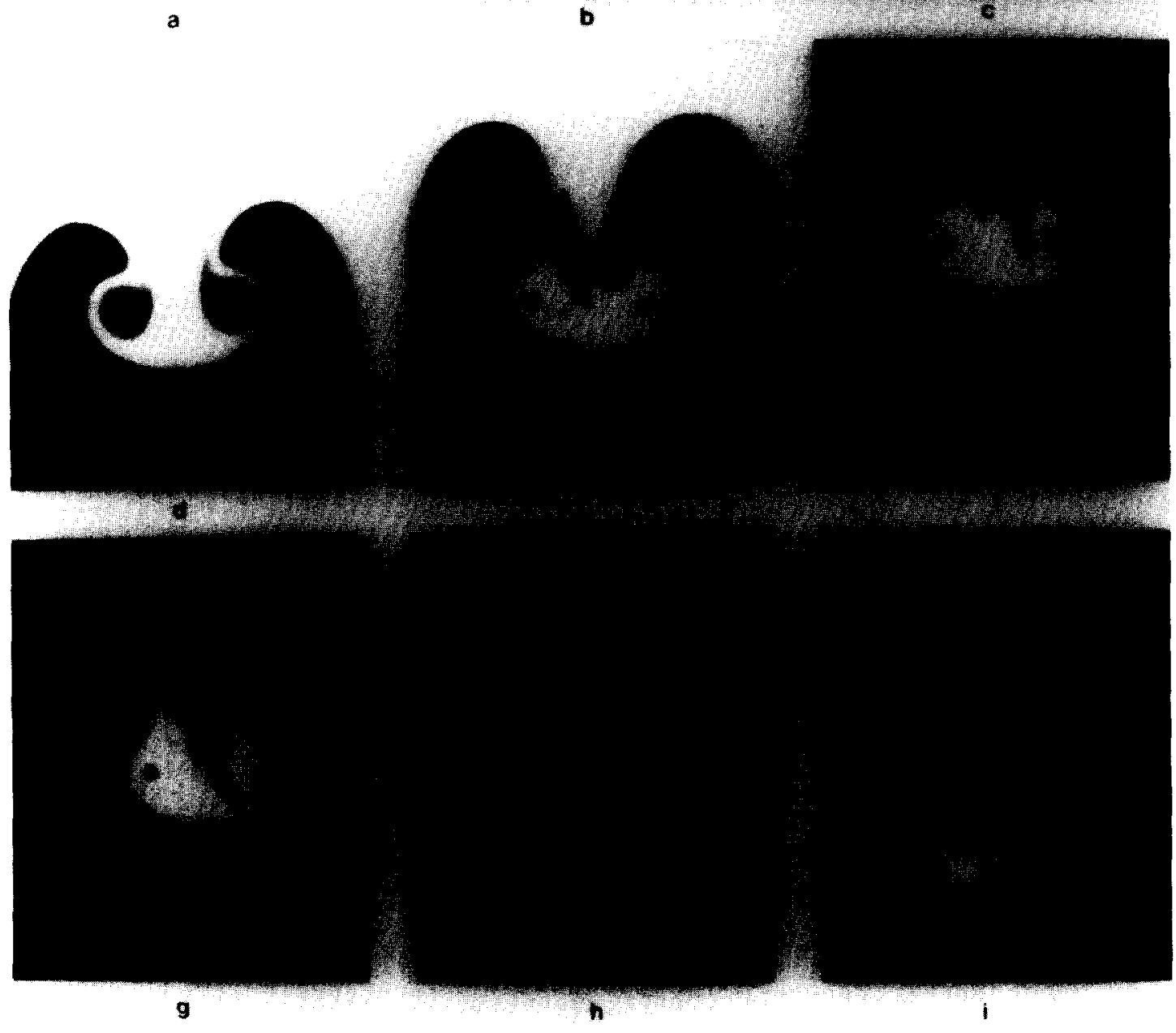

$\mathbf{g}$

h

Fig. 7. Formation of pocket of reactants with flame rollup. $U_{\theta} / S_{L}=8.8, d_{0} / \delta_{F}=37.2$, methane, $\phi=0.6$. Time: (a) $-11.4 \mathrm{~ms}$, (b) $8.6 \mathrm{~ms}$, (c) $23 \mathrm{~ms}$, (d) $34.3 \mathrm{~ms}$, (e) $57 \mathrm{~ms}$, (f) $66 \mathrm{~ms}$, (g) $71.4 \mathrm{~ms}$, (h) $77 \mathrm{~ms}$, and (i) $83 \mathrm{~ms}$.

expansion. The hot, viscous jet exiting the rear of the vortex tends to unroll the vortex, as evidenced by the white fingers of unburned reactants that extend nearly parallel to the wake.
As $U_{\theta} / S_{L}$ is increased to 12.5 the vortex undergoes transition to a turbulent vortex, as shown in Fig. 8. The flame at the vortex leading edge remains laminar and propagates slowly, if at 


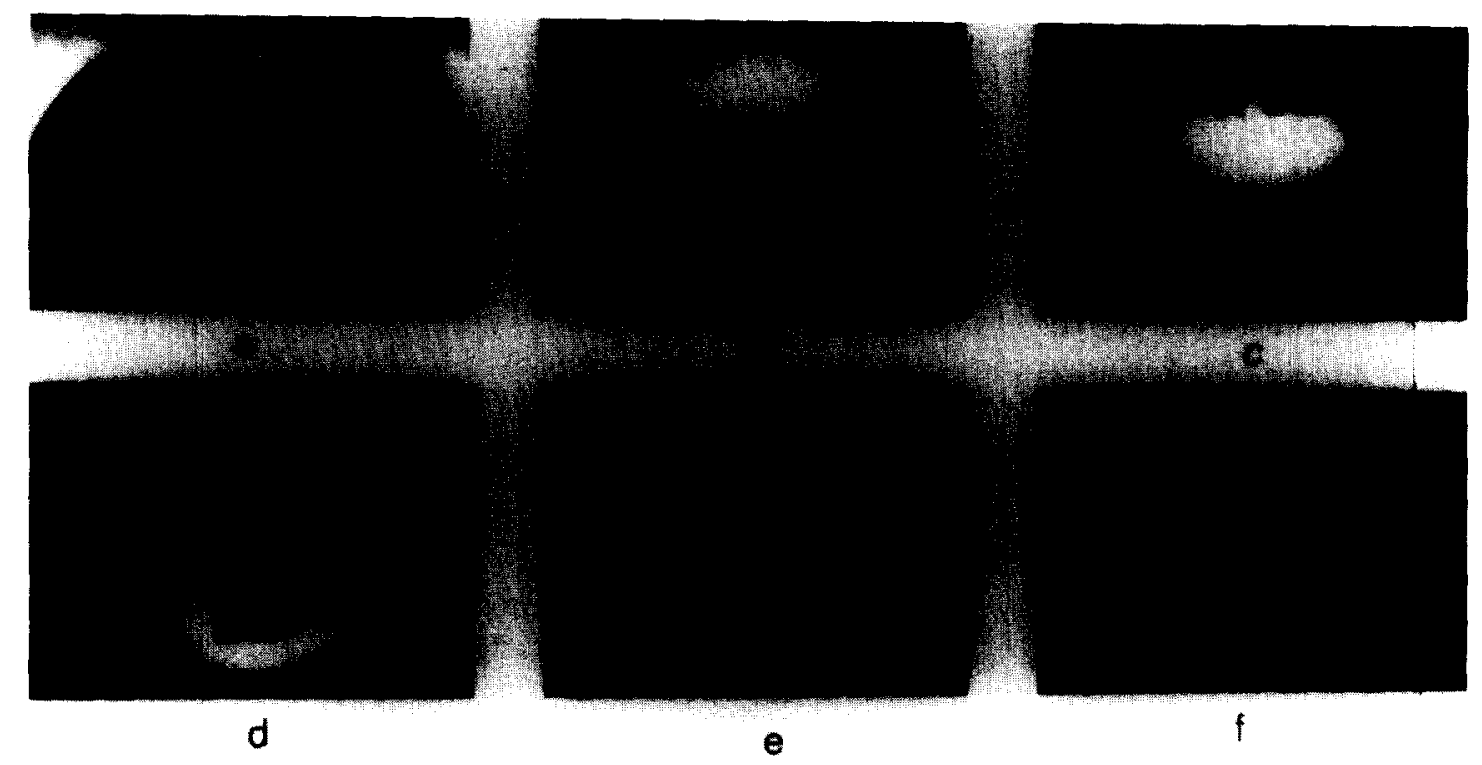

Fig. 8. Pocket of reactant formed for strongest vortex used. Rotational velocity prevents the flame near the leading edge of the vortex from propagating over the vortex, but induces the flame at the rear edge of the vortex to propagate downward into the vortex. $U_{\theta} / S_{L}=12.5, d_{0} / \delta_{F}=37.2$, methane, $\phi=0.6$. Time: (a) $42 \mathrm{~ms}$, (b) $79 \mathrm{~ms}$, (c) $96 \mathrm{~ms}$, (d) $117 \mathrm{~ms}$, (e) $133 \mathrm{~ms}$, and (f) $150 \mathrm{~ms}$.

all, but the flame at the rear of the vortex is advected forward by the rotational velocity and burns over the vortex core.

\section{REGIMES OF WRINKLING AND POCKET FORMATION}

The boundaries of the wrinkled flame regime and the pocket formation regime are plotted in Figures 9 and 10. For each data point plotted, three to five visualizations were recorded by the VCR camera; repeatability was excellent. Classical theory of flame wrinkling by Damkohler and by Shchelkin [14] predicts that the fractional increase in flame area should be only a function of normalized velocity fluctuations $\left(U_{\theta} / S_{L}\right.$ in this case) and should not depend on vortex size. These classical concepts predict that a curve defining the degree of wrinkling would be a horizontal line in Fig. 9. Instead, the boundaries between regimes in Fig. 9 have a negative slope.

The negative slope of the boundaries in Fig. 9 indicates that smaller vortices are less effective in wrinkling the flame and forming pockets than larger vortices of the same rotational velocity. Numerical simulations of Poinsot et al. [3] have predicted that the regime boundaries should have negative slope, which agrees with the present results. Smaller vortices decay more rapidly during flame passage because they have lower Reynolds number, i.e., relatively larger viscous forces than the larger vortices. In addition, smaller vortices create larger local flame curvature, which can oppose wrinkling and pocket formation due to flame stretch.

The present measurements can be used to estimate the smallest scale of turbulence that wrinkles a premixed flame, which is defined as the cutoff scale by Poinsot et al. [3]. The lower solid curve in Fig. 9 represents measurements of the smallest vortex that wrinkles the present flame, for a given vortex strength. Now, as an example, consider a hypothetical turbulent flame for which the rms velocity fluctuation $u^{\prime}$ is $75 \mathrm{~cm} / \mathrm{s}$, the integral scale $(\lambda)$ is $1.8 \mathrm{~cm}$, and the laminar burning velocity is $25 \mathrm{~cm} / \mathrm{s}$, resulting in a flame thickness of $0.022 \mathrm{~cm}$. The rightmost point on the sloped dashed line in Fig. 9 represents vortices at the integral scale for this example. The leftmost point on the dotted line represents the Kolmogorov length scale $(\eta)$ and the Kolmogorov velocity $\left(u_{K}\right)$, which are uniquely determined from the above integral scale conditions by setting $\eta$ equal to $2.3 \lambda\left(u^{\prime} \lambda / \nu\right)^{-3 / 4}$ and $u_{K}$ equal to $u^{\prime}(\eta / \lambda)^{1 / 3}$. The strengths of all turbulent vortices in this flowfield example are represented by 
the sloped dashed line. The cutoff scale that is defined in Ref. 3 is the intersection of the sloped dashed line in Fig. 9 with the measured lower boundary of the wrinkled flame regime. For the example above, the cutoff scale shown in Fig. 9 is equal to $16 \delta_{F}$ or $3.5 \mathrm{~mm}$. It can be concluded that for the above example the cutoff scale is 16 times larger than the Kolmogorov scale, which indicates that a substantial number of the smaller eddies are too weak to wrinkle the flame. Wu et al. [6] observed that the smallest wrinkles in their turbulent premixed flame also were significantly larger (i.e., 25 times larger) than their Kolmogorov scale. Wu et al. observed that increasing their turbulence level $\left(u^{\prime}\right)$ increased the strength of the smaller eddies, causing smaller scale wrinkles, which gave the flame a finegrained, fractal-like structure. This observed trend is consistent with the cutoff scale analysis of Poinsot et al. Increasing $u^{\prime}$ will increase the height of the rightmost point on the sloped dashed line in Fig. 9. Since the dashed line must maintain a slope of $1 / 3$, it is seen that the cutoff scale will decrease.

The Gibson scale $\left(\lambda_{G}\right)$ also is identified in Fig. 9 for the example conditions; $\lambda_{G}$ equals $\lambda\left(S_{L} / u^{\prime}\right)^{3}[10]$ and is seen to be six times smaller than the cutoff scale of Poinsot et al. Recent work by Wu et al. [6] and Kwon et al. [5] concludes that the Gibson scale does not represent the smallest wrinkles measured in their turbulent flame.

\section{STRETCH, CURVATURE, AND LEWIS NUMBER EFFECTS}

The Lewis number (Le) was varied so as to study both thermodiffusively unstable flames (methane-air, $\phi=0.6$ ) and diffusively stable flames (propane-air, $\phi=0.6$, Le $=1.87$ [20]). The unstable flames remained smooth and noncellular (see Figs. 4a, 6a, 7a) for $\phi<0.7$ apparently because the hydrodynamic forces associated with nonuniform gas expansion were not strong enough to trigger the wrinkling. For $0.7<\phi<1.0$, the methane-air flames were cellular and not appropriate for this type of study.

The flame regime boundaries for the diffusively unstable case (Fig. 9) and stable case (Fig. 10) have similar negative slopes. However, the vortex strengths $U_{\theta} / S_{L}$ required to form pockets in the diffusionally stable case are three times larger than the corresponding values for the unstable case. These findings indicate that flame stretch plays a significant role in the present interactions. In turbulent premixed flames, both Wu et al. [6] and Kwon et al. [5] report that preferential diffusion/flame stretch effects cause significant changes in the flame structure and the reactant consumption rates.

While the present results confirm certain trends predicted by Poinsot et al. [3], it is important to keep in mind several differences between their assumptions and the present experiment. The present vortex is an axisymmetric toroid, whereas

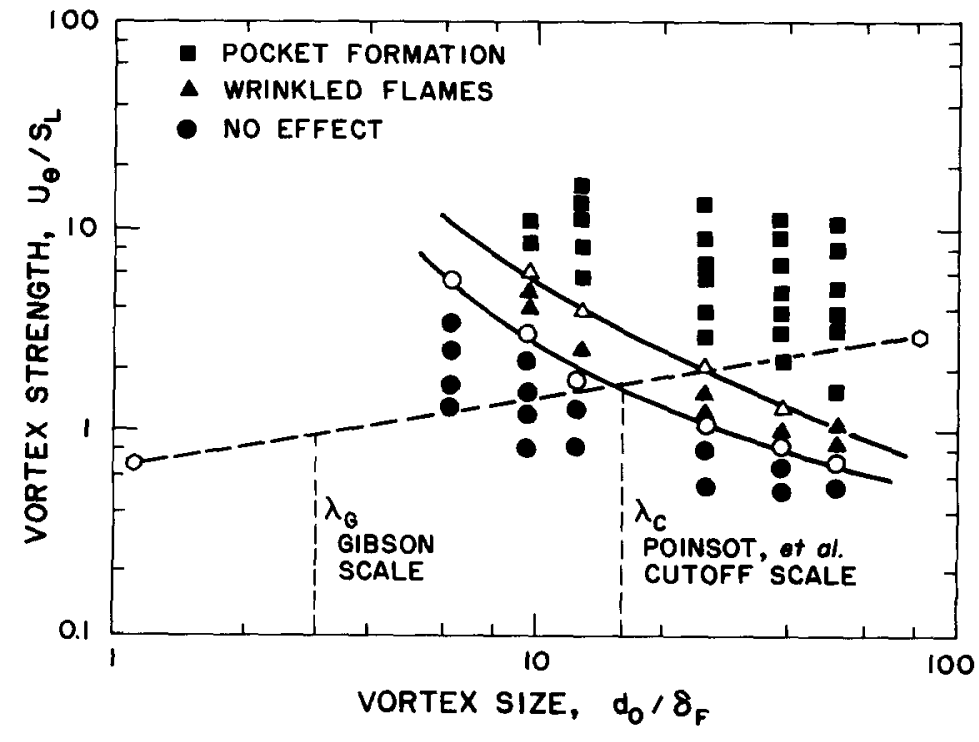

Fig. 9. Boundaries of the pocket formation/corregated flame regime (upper curve) and the minimum vortex size that wrinkles the flame (lower curve) measured for diffusionally unstable conditions. Methane, $\phi=0.6, S_{L}=8 \mathrm{~cm} / \mathrm{s}$, $\delta_{F}=0.10 \mathrm{~cm}$. Negative slope of boundaries is attributed to viscosity and flame curvature effects, as shown by numerical simulation of Poinsot, et al. [3]. Sloped dashed line indicates range of typical turbulent eddies from Kolmogorov scale to integral scale for a hypothetical flow having $u^{\prime}=75 \mathrm{~cm} / \mathrm{s}, \lambda=1.8 \mathrm{~cm}$; arrows indicate cut-off scale $\lambda_{c}$ proposed by Poinsot et al. [3] and Gibson scale $\left(\lambda_{G}\right)$ for this case. 


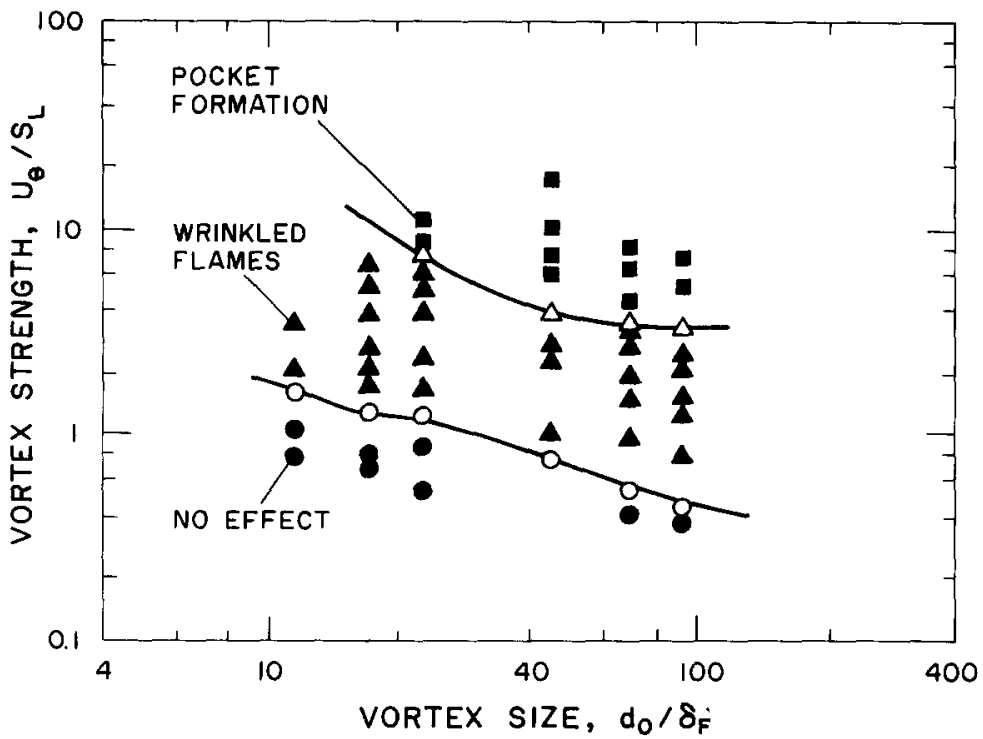

Fig. 10. Regimes of wrinkled flames and pocket formation (corrugated flames) measured for diffusionally stable conditions. Propane, $\phi=0.6, \mathrm{Le}=1.87, S_{L}$ $=14 \mathrm{~cm} / \mathrm{s}, \delta_{F}=0.055 \mathrm{~cm}$. Note that to form pockets, vortex strength must be three times larger for the above stable conditions than for unstable conditions (Fig. 9). the numerical study considers a two-dimensional pair of line vortices. The numerical study includes no vortex translational velocity or buoyancy forces which exist in the experiment. Realistic turbulent eddies will have translational velocities induced by neighboring vortices, as governed by the law of Biot and Savart [8]. The translational velocity enhances the flame wrinkling but does not, by itself, cause pockets to form.

Peters and Williams [9] have simulated the interaction of a flame with a "frozen" vortex having a velocity field that is not disturbed by the flame. For several reasons, the flame in the present experiment does not roll up and undergo three to five revolutions about the vortex core, as predicted by Peters and Williams. The potential vortex in the analysis had rotational velocities that approach an infinite magnitude near the core; the present vortex has nearly zero rotational velocity at the core center. The maximum rotational velocity in the experiment was limited by transition to turbulence. The present vortices also have a translational velocity, whereas the analysis of Peters and Williams considered the interaction to begin only after the flame overlapped the vortex core. Also, the large value of viscosity of the products and the expansion of gases that exit the vortex tend to reduce the rotational velocity; neither are considered in the constant density analysis of Peters and Williams.

The perimeter of the flame interface that is imaged in Figs. 5-8 is important because it helps to quantify the increase in reaction surface area caused by a turbulent eddy. In Fig. 11 time is normalized by $d_{0} / S_{L}$ and the measured perimeter is normalized by the unperturbed perimeter. The upper curve corresponds to an interaction in the pocket formation regime $\left(U_{\theta} / S_{L}=8.8\right)$, whereas the lower curve corresponds to an interaction at the border between the wrinkled flame regime and the pocket formation regime $\left(U_{\theta} / S_{L}\right.$ $=2.75$ ). The rapid increase in the flame perime-

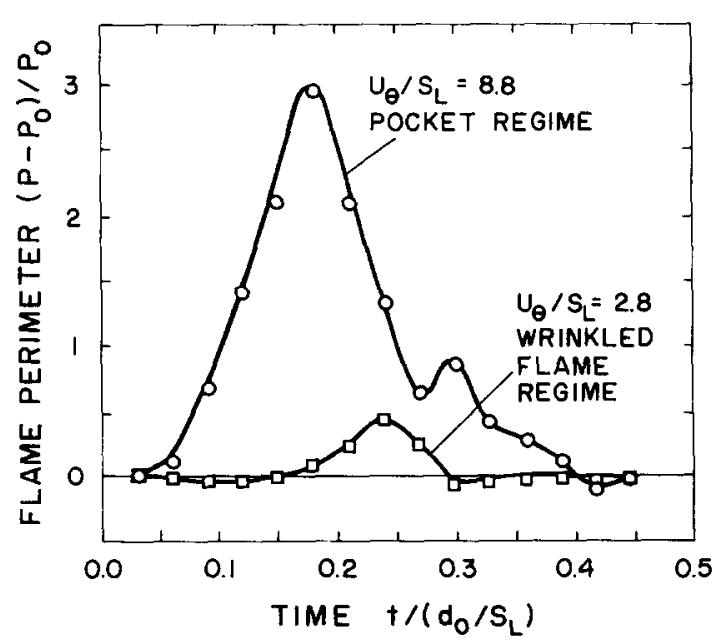

Fig. 11. Measured perimeter of laser light sheet image of flame surface. $P_{0}$ is perimeter of undisturbed flame. $d_{0}=$ $3.81 \mathrm{~cm}, S_{L}=8 \mathrm{~cm} / \mathrm{s}$, methane, $\phi=0.6$. Some predicted flame perimeters are given in Chate and Cant [7]. 
ter curve in Fig. 11 is due to the vortex translational velocity, which pulls a portion of the flame along with the vortex, and is due to some flame rollup. The later decrease in the flame perimeter is due to burnthrough of narrow regions of reactants. Increases in flame perimeter are observed to be as large as a factor of 3 , which is typical of turbulent flames. Chate and Cant [7] have calculated the flame perimeter increase for a frozen vortex velocity field and have computed trends that are similar to those shown in Fig. 11.

\section{CONCLUSIONS}

1. Three distinct regimes of the flame-vortex interaction are observed: the no-effect regime, the wrinkled flame regime, and the pocket formation regime. Boundaries between these regimes were measured. The minimum vortex size that wrinkles a premixed flame, for a given vortex strength, also was measured. Images of interactions within a fourth regime (local flame quenching) are reported elsewhere [19].

2. Smaller vortices are less likely to disturb the flame front than larger vortices having the same vortex strength $\left(U_{\theta} / S_{L}\right)$. These observations are consistent with the Navier-Stokes simulations of Poinsot et al. [3]. Smaller vortices have a lower Reynolds number than the larger vortices and thus decay due to viscous forces before they can effectively wrinkle the flame or form pockets. Smaller vortices also cause larger flame curvature, which can prevent wrinkling. Using the present results, it can be estimated that a significant fraction of the smaller vortices ahead of a typical turbulent flame are too weak to wrinkle the flame.

3. Stretch effects are observed to play an important role in wrinkling the flame (to such a degree that pockets are formed), as evidenced by large effects of Lewis number on the results.

4. The translational velocity of the vortex with respect to the reactants $\left(U_{z}\right)$ is important in the flame wrinkling and pocket formation processes. $U_{z}$ must be included for accurate numerical simulations of this experiment as well as general turbulent flows. Due to $U_{z}$ and Huyghen's principle $[6,8]$, cusps that point toward the products were formed in the flame surface; these are commonly observed in turbulent flames.

5. The flame perimeter increased by as much as a factor of 3 during vortex passage; flame perimeter subsequently decreased due to the burnthrough of narrow regions of reactants.

\section{REFERENCES}

1. Law, C. K., Twenty-Second Symposium (International) on Combustion, The Combustion Institute, Pittsburgh, 1988, pp. 1381-1402.

2. Haworth, D. C., Drake, M. C., Pope, S. B. and Blint, R. J., Twenty-Second Symposium (International) on Combustion, The Combustion Institute, Pittsburgh, 1988, pp. $589-597$

3. Poinsot, T., Veynante, D., and Candel, S., TwentyThird Symposium (International) on Combustion, The Combustion Institute, Pittsburgh, 1990, p. 613.

4. Rutland, C. J., Ferzinger, J. H., and El Tahry, S. H., Twenty-Third Symposium (International) on Combustion, The Combustion Institute, Pittsburgh, 1990, p. 621 .

5. Kwon, S., Wu, M. S., Driscoll, J. F., and Faeth, G. M., Combust. Flame, (in press).

6. Wu, M. S., Kwon, D., Driscoll, J. F., and Faeth, G. M., Combust. Sci. Technol., 78: 69-96 (1991).

7. Chate, H., and Cant, R. S., Combust. Flame, 74:1-8 (1988).

8. Ashurst, W. T., and McMurtry, P. A., Combust. Sci. Technol. 66:17-38 (1989).

9. Peters, N., and Williams, F. A., Twenty-Second Symposium (International) on Combustion, The Combustion Institute, Pittsburgh, 1988, pp. 495-503.

10. Peters, N., Twenty-First Symposium (International) on Combustion, The Combustion Institute, Pittsburgh, 1986, pp. $1231-1250$.

11. Dixon-Lewis, G., Fourth Symposium (International) on Combustion, The Combustion Institute, Pittsburgh, 1952, pp. 263-270.

12. Yamaoka, I., and Tsuji, H., Twentieth Symposium (International) on Combustion, The Combustion Institute, Pittsburgh, 1984, pp. 1883-1892.

13. Yu, G., Law, C. K., and Wu, C. K., Combust. Flame 63:339-347 (1986).

14. Lewis, B., and von Elbe, G., Combustion, Flames and Explosions of Gases. Academic, New York, 1961

15. Jarosinski, J., Lee, J. H. S., and Knystautas, R., Twenty-Second Symposium (International) on Combustion, The Combustion Institute, Pittsburgh, 1988, pp. 505-514.

16. Maxworthy, T., J. Fluid Mech. 51:15-32 (1972).

17. Dahm, W. J. A., Scheil, C. M., and Tryggvason, G., J. Fluid Mech. 205:1-43 (1989).

18. MacGarvey, R. H., and MacLatchy, C. S., Can. J. Phys. 42:678-683 (1963).

19. Roberts, W. L., Driscoll, J. F., Drake, M. C., and 
Ratcliffe, J., Eastern States Section Meeting of the Combustion Institute, Ithaca, NY, October 1991.

20. Mizomoto, M., and Yoshida, H., Combust. Flame 70:47-60 (1987).

Received 12 May 1991; revised 8 August 1991

\section{APPENDIX - MEASURED VORTEX VELOCITY FIELD}

Velocity profiles were measured using laser velocimetry to characterize the strength of each vortex used and to provide input to future numerical models. $U_{\theta}$ is defined as the maximum rotational velocity in the undisturbed vortex. Using conservation of momentum and mass relations [16], $U_{\theta}$ is predicted to be proportional to $(\Delta V)^{2} / t_{r} d_{0}{ }^{5}$. The amount of fluid pushed through the orifice by the loudspeaker is $\Delta V$, which is proportional to the voltage applied across the speaker coils, $t_{r}$ is the time it takes this voltage to reach its final value, and $d_{0}$ is the diameter of the orifice, all three of which are variables in this experiment. In addition, the analysis indicates that $U_{z}$ should be nearly equal to $U_{\theta}$. A series of calibration curves were generated that relate the measured value of $U_{\theta}$ and $U_{z}$ to the operating parameter $(\Delta V)^{2} / t_{r} d_{0}{ }^{5}$.

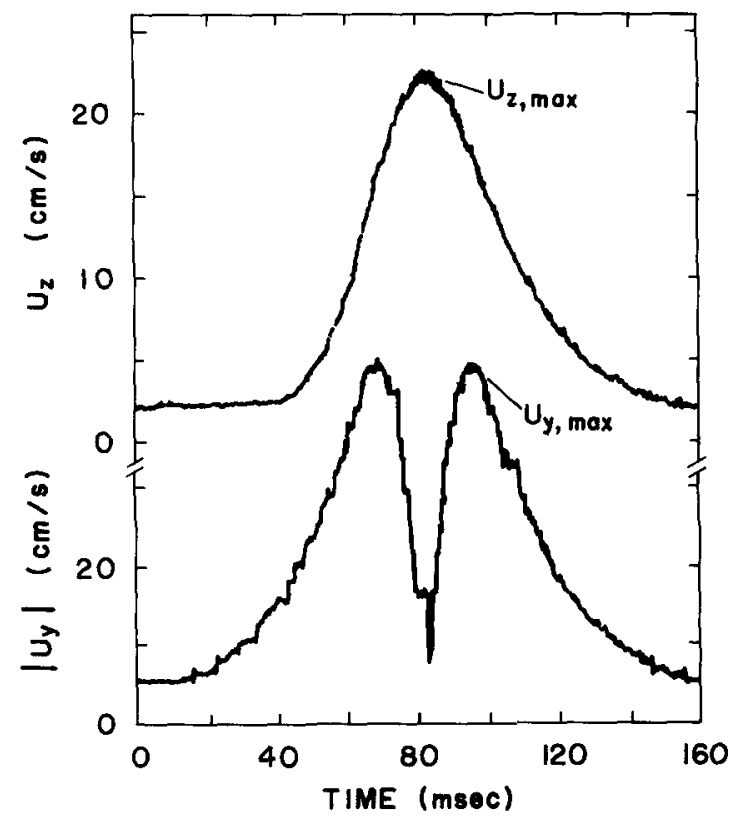

Fig. 12. Typical time history of axial velocity $\left(U_{z}\right)$ and transverse velocity $\left(U_{y}\right)$ as vortex passes over laser velocimeter measurement location. Lower curve indicates that rotational velocity profile is similar to a Rankine vortex.
All velocities were measured at the same downstream location, at a point where the flame would first interact with the vortex. Both $U_{z}$ and $U_{y}$ were measured as functions of time and $y$ location, where $z$ is the axial direction of vortex motion (Fig. 2) and $y$ is perpendicular to $z$. A single component LDV system was operated in the forward scattering mode and used a commercial counter processor. An analog output relating voltage to velocity was plotted as the vortex propagated over the probe volume. Typical plots of velocity versus time are shown in Fig. 12.

To define the core of the vortex, $U_{z, \max }$ was defined as the maximum value of the profile of $U_{z}$ versus time, as shown in the upper curve in Fig. 12. At each $y$ location, a value of $U_{z, \max }$ was determined and is plotted in the lower curve of Fig. 13. The lower curve in Fig. 13 is the axial

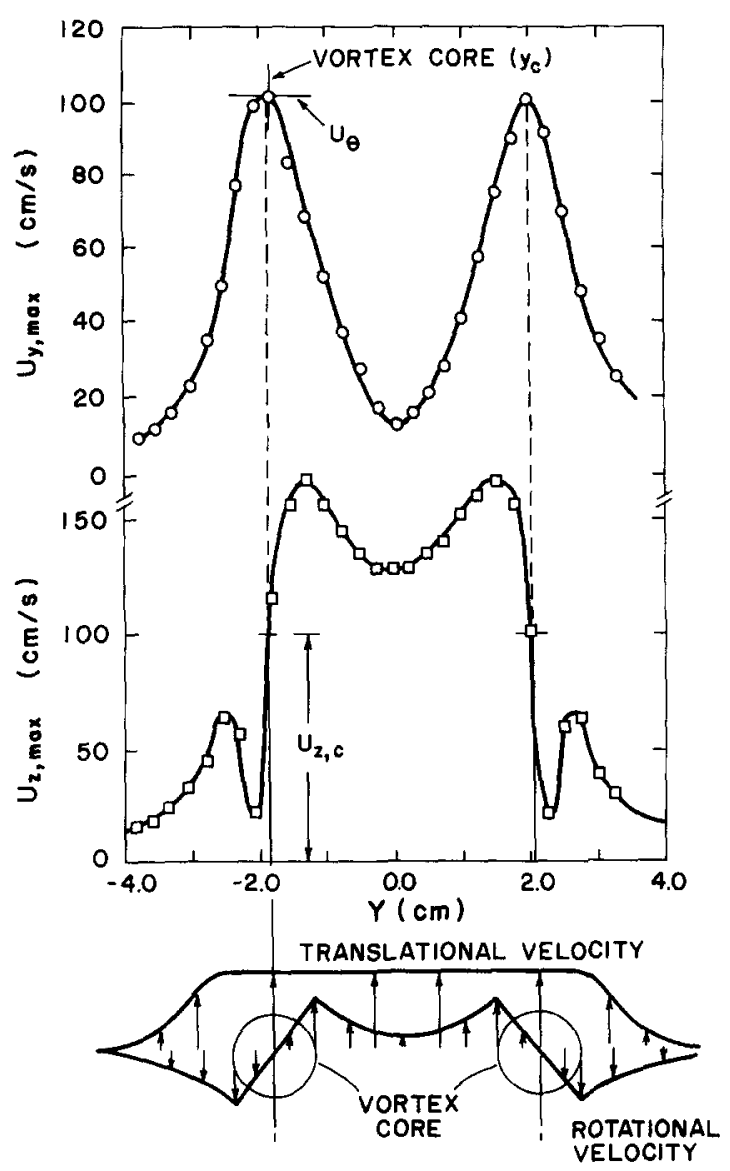

Fig. 13. Radial variation of $U_{y, \max }$ and $U_{z, \max }$, as defined in Fig. 12, used to determine core location, maximum rotational velocity $U_{\theta}$, and axial velocity of core $U_{z, c}$ from laser velocimeter data. $U_{\theta}=100 \mathrm{~cm} / \mathrm{s}, d_{0}=3.81 \mathrm{~cm}$. 
velocity along the $y$ axis, i.e., along the line drawn between the vortex cores. On centerline $(y=0), U_{z, \max }$ is about $130 \mathrm{~cm} / \mathrm{s}$ due to the superposition of induced velocities from each of the core sections shown in Fig. 13. At $y=1.5$ $\mathrm{cm}, U_{z, \max }$ reaches a maximum where the rotational velocity about the core is maximum. At $y=2.2 \mathrm{~cm}, U_{z, \max }$ has decreased to a local minimum of $20 \mathrm{~cm} / \mathrm{s}$, which is the sum of the positive translational velocity of the core $(100$ $\mathrm{cm} / \mathrm{s}$ ) and the negative rotational velocity. The core is defined as the location halfway between the maximum value of $U_{z, \max }$ at $y=1.5 \mathrm{~cm}$ and the local minimum at $y=2.2 \mathrm{~cm}$.

The quantity $U_{\theta}$ is defined as the maximum rotational velocity within the vortex and was determined as follows. The maximum value of the profile of $U_{y}$ versus time from the lower plot in Fig. 12 is defined as $U_{y, \max } . U_{y, \max }$ is plotted as a function of $y$ in the upper plot of Fig. 13. At the core location, the value of $U_{y, \max }$ is due only to rotation, and thus $U_{\theta}$ is defined as $U_{y, \max }$ at the core location ( $y=y_{c}$, as determined above). Figure 14 is a plot of the core translational velocity $U_{z, c}$ and $U_{\theta}$ as a function of the experiment parameters. Figure 14 shows that as speaker voltage ramp time $t_{r}$ is varied, both $U_{\theta}$ and $U_{z}$ are

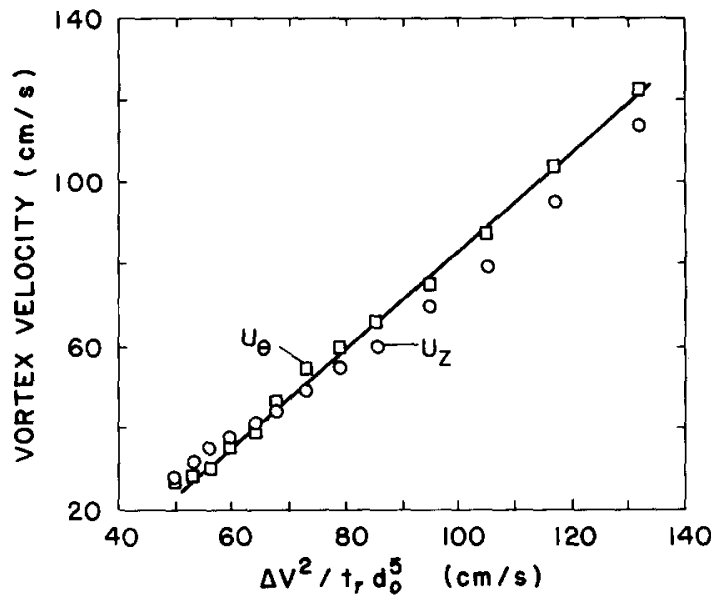

Fig. 14. Measured maximum rotational velocity $\left(U_{\theta}\right)$ of vortex and axial velocity of core $\left(U_{z, c}\right)$ for a given loudspeaker volume displacement $(\Delta V)$, voltage ramp-up time $\left(t_{r}\right)$, and orifice diameter $\left(d_{0}\right)$. Values are determined from laser velocimeter data as typified by Fig. 12 and 13.

directly proportional to $(\Delta V)^{2} / t_{r} d_{0}{ }^{5}$, as predicted by conservation of momentum concepts. However, as $\Delta V$ and $d_{0}$ are varied, the agreement with analysis is not as good as shown in Fig. 14. In all cases, however, the rotational and translational velocities are nearly equal, within experimental error. 